\title{
Semantic priming and schizotypal personality: reassessing the link between thought disorder and enhanced spreading of semantic activation
}

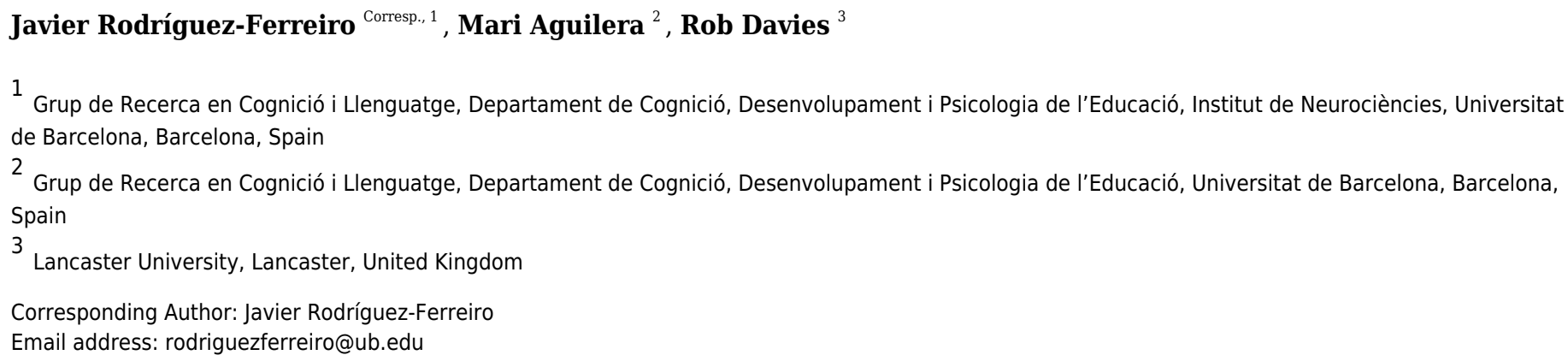

The term schizotypy refers to a group of stable personality traits with attributes similar to symptoms of schizophrenia, usually classified in terms of positive, negative or cognitive disorganization symptoms. The observation of increased spreading of semantic activation in individuals with schizotypal traits has led to the hypothesis that thought disorder, one of the characteristics of cognitive disorganization, stems from semantic disturbances. Nevertheless, it is still not clear under which specific circumstances (i.e. automatic or controlled processing, direct or indirect semantic relation) schizotypy affects semantic priming or whether it does affect it at all. We conducted two semantic priming studies with volunteers varying in schizotypy, one with directly related prime-target pairs and another with indirectly related pairs. Our participants completed a lexical decision task with related and unrelated pairs presented at short $(250 \mathrm{~ms})$ and long $(750 \mathrm{~ms})$ stimulus onset asynchronies (SOAs). Then, they responded to the brief versions of the Schizotypal Personality Questionnaire and the Oxford-Liverpool Inventory of Feelings and Experiences, both of which include measures of cognitive disorganization. Bayesian mixed-effects models indicated expected effects of SOA and semantic relatedness, as well as an interaction between relatedness and directness (greater priming effects for directly related pairs). Even though our analyses demonstrated good sensitivity, we observed no influence of cognitive disorganization over semantic priming. Our study provides no compelling evidence that schizotypal symptoms, specifically those associated with the cognitive disorganization dimension, are rooted in an increased spreading of semantic activation in priming tasks. 


\section{Semantic priming and schizotypal personality:}

2 reassessing the link between thought disorder and

3 enhanced spreading of semantic activation

4 Javier Rodríguez-Ferreiro ${ }^{1}$, Mari Aguilera ${ }^{2}$, Rob Davies ${ }^{3}$

$5{ }^{1}$ Grup de Recerca en Cognició i Llenguatge, Departament de Cognició, Desenvolupament i

6 Psicologia de l'Educació, Institut de Neurociències, Universitat de Barcelona, Barcelona, Spain

$7{ }^{2}$ Grup de Recerca en Cognició i Llenguatge, Departament de Cognició, Desenvolupament i

8 Psicologia de l'Educació, Universitat de Barcelona, Barcelona, Spain

$9{ }^{3}$ Lancaster University, Lancaster, United Kingdom

11 Corresponding Author:

12 Javier Rodríguez-Ferreiro

13 Campus Mundet, psg de la Vall d'Hebron, 171, Barcelona, 08035, Spain

14 Email address: rodriguezferreiro@ub.edu 


\section{Abstract}

17 The term schizotypy refers to a group of stable personality traits with attributes similar to 18 symptoms of schizophrenia, usually classified in terms of positive, negative or cognitive

19 disorganization symptoms. The observation of increased spreading of semantic activation in individuals with schizotypal traits has led to the hypothesis that thought disorder, one of the characteristics of cognitive disorganization, stems from semantic disturbances. Nevertheless, it is still not clear under which specific circumstances (i.e. automatic or controlled processing, direct or indirect semantic relation) schizotypy affects semantic priming or whether it does affect it at all. We conducted two semantic priming studies with volunteers varying in schizotypy, one with directly related prime-target pairs and another with indirectly related pairs. Our participants completed a lexical decision task with related and unrelated pairs presented at short $(250 \mathrm{~ms})$ and long $(750 \mathrm{~ms})$ stimulus onset asynchronies (SOAs). Then, they responded to the brief versions of the Schizotypal Personality Questionnaire and the Oxford-Liverpool Inventory of Feelings and Experiences, both of which include measures of cognitive disorganization. Bayesian mixedeffects models indicated expected effects of SOA and semantic relatedness, as well as an interaction between relatedness and directness (greater priming effects for directly related pairs). Even though our analyses demonstrated good sensitivity, we observed no influence of cognitive disorganization over semantic priming. Our study provides no compelling evidence that schizotypal symptoms, specifically those associated with the cognitive disorganization dimension, are rooted in an increased spreading of semantic activation in priming tasks. 


\section{Introduction}

38 In the influential model proposed by Meehl (1962), schizotypy refers to a personality

39 organization which stems from a pattern of brain functioning that can lead to the development of 40 schizophrenia, given interactions with differences in genetic or social conditions. Schizotypal

41 personality is characterized by a group of stable personality traits expressed in behaviours similar 42 to symptoms of schizophrenia, encompassing positive (hallucinations, paranoia, magical 43 thinking...), negative (anhedonia, avolition...) and disorganized (odd speech and behavior, 44 thought disorder...) symptoms (Raine \& Benishaw, 1995) that can be present in the sub-clinical 45 population (see, also, Ettinger et al., 2014; Kwapil \& Barrantes-Vidal, 2015). Thought disorder 46 (cognitive slippage or associative loosening) is one of the fundamental symptoms of schizotypy 47 (Meehl, 1962). This characteristic, more specifically referred to as formal thought disorder (as 48 opposed to disorders of thought content, such as delusions), has been proposed to stem from 49 atypical patterns of semantic activation (Spitzer, 1997). This hypothesis is based on findings 50

Schizophrenic thinking is often characterized by derailment or loosening of associations between ideas, as well as by obliquely related association or tangentiality (Rossell et al., 2000; Spitzer, 1997). The intrusion of these oblique or unusual associations into the speech of patients with schizophrenia has been attributed to enhanced distribution of semantic activation during lexical access (Spitzer, 1997). This hypothesis is based on a model of semantic memory assuming that words are represented as interconnected nodes which are activated for utterance, and that each time one of the nodes is activated, the activation spreads to related nodes, lowering their threshold for subsequent activation (Collins \& Loftus, 1975). The problem is that the ways in which differences in schizotypal personality traits are related to differences in semantic processing have been inconsistently observed. We present an investigation comprising two studies designed to clarify the scope of semantic priming, as a marker of enhanced semantic activation, given differences in schizotypal personality traits.

The idea that schizotypal individuals might present enhanced spreading of semantic activation is of ongoing interest because such enhanced activation has been related to possible links between 
68 higher levels of schizotypal these personality traits and healthy or even favorable functioning, 69 especially in relation to creativity. This hypothesis parallels the possible association between 70 schizophrenia and creativity, which has been suggested to be specifically mediated by the presence of thought disorder (Barrantes-Vidal, 2004; Hasenfus \& Magaro, 1976). In this sense,

72 the generation of uncommon solutions to a given problem by creative individuals could be 73 related to the capacity to activate remote semantic information within the semantic network

74 (Mohr \& Claridge, 2015). Thus, previous studies have observed increased originality of the 75 76 responses in semantic fluency tasks (Kiang \& Kutas, 2006; Minor \& Cohen, 2012) for volunteers with higher levels of schizotypal traits. These observations can be interpreted to reflect

77 78 79 80 heightened spreading of semantic activation through the semantic system, which could explain the production of infrequent (original) responses in fluency tasks due to the activation of farther nodes in the semantic network.

In the same vein, previous research has shown that individuals with higher scores on schizotypy scales tend to present higher false memory response rates in response to unpresented critical words, having previously been exposed to lists of words semantically related to them (Laws \& Bhatt, 2005; Saunders et al., 2012). As with the production of more original responses in fluency tasks, increased false memory responses could be argued to result from enhanced spreading of activation from stimulus words to unpresented but semantically related critical words, a transmission of activation that is sufficient to trigger false positives in response to recall or recognition tests. Crucially, in accordance with previous observations with schizophrenia patients (see Pomarol-Clotet et al., 2008), some authors argue that this mechanism could also underlie the presence of atypical speech patterns or ideas of reference associating unrelated events in schizotypal individuals (Mohr et al., 2001; Pizzagalli et al., 2001). Nevertheless, other studies have failed to obtain significant associations between the originality of fluency responses (Hori et al., 2008; Rodríguez-Ferreiro \& Aguilera, 2019) or semantic-based false memory rates (Corlett et al., 2009; Kanemoto et al., 2013; Rodríguez-Ferreiro et al., 2020) and differences on schizotypal traits, so the reliability of these observations is unclear.

Several studies have focused on the possible relation between schizotypal personality and semantic priming. As noted by Spitzer (1997), semantic priming might be the most 
99 straightforward measure of enhanced (faster and further-reaching) spreading of semantic

100 activation in relation to thought disorder in schizophrenia. The semantic priming effect (Lucas,

101 2000; Meyer \& Schvaneveldt, 1971) refers to the tendency to respond faster when a target word

102 (e.g. bread) is preceded by a semantically related prime (e.g. butter), compared to an unrelated

103 prime (e.g. doctor) during a speeded language task such as lexical decision (i.e. deciding whether

104 a given letter-string corresponds to a real word or not). This paradigm is relevant for the study of

105 semantic processing because it is assumed to inform us about the process of spreading of

106 semantic activation between related nodes in the semantic system: responses to target words

107 presented after related primes are faster because the semantic activation of the prime has spread

108 through the system, pre-activating related words such as the target (Yap et al., 2017).

109

110 In a meta-analysis of the results from 36 studies of semantic priming in schizophrenia patients,

111 Pomarol-Clotet et al. (2008) found qualified evidence for an increase in semantic priming in

112 patients with thought disorder compared to non-schizophrenic controls. However, the specificity

113 of priming differences was found to be unclear, with patients with thought disorder showing no

114 increase in priming compared to patients without thought disorder. In addition, the meta-analysis

115 could not exclude the possibility that increased semantic priming in patients was an artefact of a

116 general slowing of reaction times in schizophrenia. These qualifications in the clarity of the

117 evidence warrant further investigation of the nature of semantic priming in schizophrenia. A

118 review of the literature reveals a lack of clarity, also, in otherwise intriguing results from

119 research on the association between differences in schizotypal personality traits and differences

120 in semantic priming.

121

122 In Table 1 we summarize the findings of seven studies which have explored the possible relation 123 between schizotypal personality and semantic priming effects. Depending on their aims and 124 initial hypotheses, each study has applied a slightly different experimental approach. Researchers 125 have either used comparisons between priming effects observed in high and low scorers on 126 schizotypal personality scales, or have analysed correlations between schizotypy scores and 127 priming effects. The most frequent tools applied to the assessment of schizotypal traits are the 128 Schizotypal Personality Questionnaire (SPQ, Raine, 1991) and the Oxford Life Inventory of 129 Feelings and Experiences (O-LIFE, Mason \& Claridge, 2006). The SPQ was designed to assess 
130 the DSM-III-R symptoms of Schizotypal Personality Disorder (American Psychiatric

131 Association, 1987) and comprises nine subscales loading onto three factors: cognitive-perceptual

132 or positive schizotypy (unusual perceptual experiences, ideas of reference, magical thinking and

133 paranoid ideation); interpersonal or negative schizotypy (social anxiety, no close friends,

134 constricted affect and paranoid ideation); and disorganization (odd behavior and appearance, odd

135 speech). In contrast, the O-LIFE distinguishes between four dimensions: unusual experiences

136 (positive dimension: perceptual aberrations, magical thinking and hallucinations); introvertive

137 anhedonia (negative dimension); cognitive disorganization (disorganized dimension: poor

138 attention, poor decision making, social anxiety and disorganized speech); and impulsive

139 nonconformity, which refers to unstable mood, antisocial behavior and lack of control. The

140 relevance of this last dimension to the schizotypy construct has been disputed and it is not

141 included in questionnaires based on three-factor solutions of the schizotypy construct like the

142 SPQ. The scores obtained in the disorganized dimensions of these questionnaires have been

143 interpreted to reflect the presence of thought disorder in the volunteers (Johnston et al., 2008;

144 Neill et al., 2014; Rossell et al., 2014). Although it could be argued that the disorganization

145 dimension of SPQ focuses more on thought disorder than the analogous cognitive

146 disorganization dimension of O-LIFE, this second questionnaire has been used more often in the

147 literature studying the relation between schizotypy and semantic processing.

148

149 One key aspect of these studies was the examination of whether the association between thought

150 disorder and semantic memory relies on automatic processes (i.e. depending on automatic

151 spreading of semantic activation as suggested by Spitzer, 1997) or on controlled processes. In

152 relation to this second possibility, some authors have argued that language disturbances in

153 individuals with schizophrenia are associated with attentionally-driven (controlled) processes

154 (Callaway \& Naghdi, 1982). These processes (see Neely \& Keefe, 1989 for an extended

155 discussion) include expectancy effects (i.e. pre-lexical generation of potential targets after the

156 presentation of the prime) and semantic matching effects (i.e. retrospective matching of the

157 target with the prime due to their semantic relation). In order to distinguish between automatic

158 and controlled processes, researchers have manipulated the stimulus onset asynchrony (SOA)

159 between primes and targets. Thus, it is assumed that priming effects obtained with short SOAs, 
160 around $250 \mathrm{~ms}$, reflect automatic processing, whereas effects obtained with longer SOAs, longer

161 than $700 \mathrm{~ms}$, are indicative of controlled processing (Morgan et al., 2006).

162

163 A second critical variation among studies has involved the manipulation of the semantic distance

164 between primes and targets. Participants can either be presented with directly related (e.g. lion -

165 tiger) or indirectly related stimulus pairs (e.g. lion - stripes, related through tiger). Following

166 Spitzer's model (1997), and given the association between thought disorder and schizotypy, if

167 thought disorder is rooted in enhanced spreading of activation in the semantic system then we

168 should expect that individuals obtaining high scores on schizotypal personality scales should

169 show greater direct semantic priming. The impact of individual differences on schizotypal scales,

170 especially, on disorganization, should be still more prominent in indirect semantic priming

171 because the presence of faster and further-reaching activation spreading among higher scoring

172 individuals should facilitate the connection between indirectly related pairs of words relative to

173 lower scoring individuals.

174

175 In regard to direct semantic priming, Moritz et al. (1999) observed greater effects of prime-target

176 relatedness for individuals who scored high on the language subscale of the Frankfurt Complaint

177 Questionnaire (Süllwold, 1991), given directly related primes both at short and at long SOAs.

178 This result was partially replicated by Neil et al. (2014) in a study assessing only short SOA

179 conditions: they found that individuals scoring high on disorganization showed increased direct

180 priming. In contrast, Moritz et al. (1999) found no significant differences in priming effects

181 when comparing participants distinguished according to SPQ scores. Further, in studies

182 recording either global O-LIFE scores (Morgan et al., 2006) or scores on the O-LIFE

183 disorganized subscale (Johnston et al., 2008; Rossell et al., 2014; Tan \& Rossell, 2017), no

184 correlation was observed between direct priming effects and individual differences in schizotypy 185 traits.

186

187 In regard to indirect semantic priming, Moritz et al. (1999) observed greater priming effects for

188 higher scorers on the language subscale of the Frankfurt Complaint Questionnaire (Süllwold, 189 1991) at short SOA but not at long SOA. (As noted, they reported no differences in priming 190 when comparing individuals varying on the SPQ.) Johnston et al. (2008) reported a significant 
191 positive correlation between scores on O-LIFE cognitive disorganization and indirect priming 192 effects at short SOA. However, in contrast, Neill, Rossell and Kordzadze (2014) observed the 193 opposite: participants who scored high on O-LIFE disorganization showed reduced -- not 194 increased -- indirect priming effects. Finally, Rossell, Chong, O’Connor and Gleeson (2014) 195 observed a significant positive correlation between scores in the O-LIFE disorganized dimension 196 and indirect priming effects at long SOA.

197

198

199

200

201

202

203

204

205

206

207

208

209

210

211

212

213

214

215

216

217

218

219

220

Although they do not derive from a reaction time study, we should comment also on the findings reported by Kiang, Prugh and Kutas (2010). Their aim was to assess the possible influence of schizotypal traits on the amplitude of the N400 component, a relatively negative electrocortical component which peaks approximately $400 \mathrm{~ms}$ after the appearance of meaningful stimuli like words or pictures. Global SPQ scores were negatively correlated with indirect priming effects on the N400 signal at both short and long SOAs (where priming was observed as differences in N400 amplitudes). Scores on the positive dimension were negatively correlated with direct priming effects at both SOAs, and with indirect priming effects at the shorter SOA only.

These studies present evidence that there are, potentially, associations between differences in schizotypal personality and differences in semantic activation, as reflected in semantic priming effects. However, it is unclear which dimensions of schizotypal personality correlate to semantic priming, and whether a relation between differences in schizotypal personality traits and in priming effects are evident for directly or indirectly related prime-target stimuli, at short or at long SOAs. This lack of clarity in the evidence base is important because the potential association between differences in schizotypal personality and differences in semantic activation is critical to theoretical accounts of schizotypy-related behaviours observed in the sub-clinical population, based in the assumption of enhanced spreading activation. Moreover, it is important because there is also suggestive evidence (also qualified) which relates differences in schizophrenia to differences in semantic priming, and this evidence has similar theoretical implications. What our review of studies on schizotypal personality and priming shows is that further investigation is required to examine trait differences comprehensively across measures of the dimensions of schizotypal personality, and to examine how such trait differences relate to 
221 variation in semantic priming under different conditions of relatedness and stimulus onset

222 asynchrony. We report such an investigation.

223

224 While the results of these previous studies are interesting and, of course, have important

225 implications, a close review of the methods suggest strong grounds for expecting that some of

226 the inconsistency among findings may stem from the influence of random variation in samples.

227 This is because the methodological issues that concern us will tend to reduce the sensitivity or

228 precision of analyses and such limitations will tend to afford more space for random variation to 229 have an impact.

230

231 Our first concern relates to the selection of sub-groups from participant samples (e.g. Morgan et 232 al., 2006; Moritz et al., 1999; Neill et al., 2014) which implies dichotomizing a continuous 233 variable (individual differences on a schizotypy dimension). The problem with this approach is 234 that it has been shown that the practice results in underestimates of effect sizes, reducing the 235 sensitivity or power of statistical hypothesis tests (Cohen, 1983). We note, also, that there has 236 been a tendency for studies in the field to involve relatively small participant samples (about 35 23760 participants) with few exceptions (e.g., Moritz et al., 1999), again limiting the sensitivity of 238 statistical tests. We would argue that what is required is an investigation that directly examines 239 the ways in which individual differences in schizotypy, across a reasonably sized participant 240 sample, interact with indicators of semantic processing.

242 A further, potentially critical, methodological limitation is the use of difference scores,

243 calculated as an index of semantic processing per individual, to examine the relationship between 244 semantic processing and variation on schizotypal dimensions. Researchers have, for example, 245 calculated the difference between the mean RT of responses under unrelated compared to related 246 priming conditions, calculating the difference score in order to gauge the priming effect per 247 person (Johnston et al., 2008; Morgan et al., 2006; Moritz et al., 1999; Neill et al., 2014; Tan \& 248 Rossell, 2017), or event-related potentials signal amplitude per condition (Kiang et al., 2010) so 249 that it is then possible to analyse the correlation between the priming effect difference scores and 250 scores on schizotypy scales. The results of such analyses will have important limitations because 251 within-subjects difference scores are notoriously unreliable (as discussed, e.g., by Kliegl et al., 
252 2010). This unreliability can be understood to result from the implication of the variance sum 253 law (Howell, 2016): the variance of the difference of two variables is the sum of their variances 254 (minus the correlation between the variables). This tells us that the variance of the difference 255 score will, in effect, equal the sum of the variance of the mean RT in one condition plus the 256 variance of the mean RT in the other condition. Given that variance about the mean indicates the 257 reliability of estimates so that means associated with wider variances are known to be less 258 reliable, the reduced reliability will limit the sensitivity of analyses. This is because the 259 reliabilities of two measures provide an upper bound on the possible correlation that can be 260 observed between the two measures (Vul et al., 2009).

261

262 The key problem for researchers seeking to draw conclusions from previous observations is that 263 when power (or sensitivity) is low, studies that show statistical significance will tend to be at risk 264 of presenting exaggerated estimates. Vasishth, Mertzen, Jäger and Gelman (2018) present analyses which demonstrate that in low-power scenarios the estimates from repeated samples will fluctuate around the true value, potentially giving rise to errors both in the estimation of the magnitude of the effect and in the estimation of the sign of the effect (Gelman \& Carlin, 2014). The overestimates will occur because the standard error is relatively large in low-power situations. The consequence of broadly spread sampling distributions of the mean (wider because of larger standard errors) is that there is then a greater chance that extreme values are observed. In the present investigation, we eschewed the use of traditional null hypothesis significance testing (we adopt Bayesian methods) in part because of these problems but also to focus our observations on the estimated magnitude and sign of the effects of interest.

275 An alternate way of looking at the analysis problem is that we must address it as efficiently as 276 possible with respect to the use of the data collected in test samples. We are seeking to identify 277 the ways in which semantic priming may vary in association with individual differences in 278 schizotypal dimensions. Calculating difference scores per person corresponds to no-pooling 279 analyses in which the per-person difference score identifies the priming for that person, this 280 leaves us prone to using less reliable estimates of priming (based on the data of just that person) 281 and, critically, it ignores what we can find out about other participants (i.e., the average priming 282 effect across the participant sample). Of course, individuals will vary so we cannot, either, seek 
283 to examine the correlation between the priming effect over all participants and variation in 284 schizotypy scores (the complete-pooling approach) because that would ignore the individual 285 differences that can be presumed to be present in the data. What is required is a method of 286 analysis that allows us to estimate the average (or population-level) effects of experimental 287 factors like semantic relatedness, while taking into account the random as well as the systematic 288 variation between individuals tested in our study. That method is provided in multilevel or

289 290

291

292

293

294

295

296

297

298

299

300

301

302

303

304

305

306

307

308

309

310

311

312

313 mixed-effects modelling (Gelman \& Hill, 2007), and that is the method we use in our analyses.

How, then, can we examine how semantic processing varies among individuals varying in schizotypy? Our approach is to consider the impact of interactions between the effects of semantic prime-target relatedness, or the directness of relatedness, and the effect of variation in schizotypal dimension. If the question is: how do individuals varying on a schizotypal dimension vary in how they process semantics? -- we can frame the means to address it by supposing that semantic processing can be gauged in terms of the effect of relatedness, the average difference between related and unrelated primes. If we are seeking to test how that difference is, itself, different among individuals at different levels of schizotypy then we are, by definition, seeking to test the interaction between the effect of prime-target relatedness and the effect of schizotypy (Cohen et al., 2003) because we are examining how the effect of one variable (relatedness) can vary at different levels of a second variable (schizotypy). This is the approach we take in our investigation.

All in all, although the idea that schizotypal personality is associated with semantic disturbances is commonly espoused, it remains unclear under which specific circumstances schizotypy affects semantic priming or whether it does affect it at all. For this reason, some authors have stressed the need to replicate previous studies in order to clarify the nature of the association between schizotypy and priming effects (Rossell et al., 2014). We present the results of two studies assessing the association between schizotypy and semantic processing. Given the focus of previous studies on thought disorder, we hypothesized, firstly, that cognitive disorganization would be positively related to semantic priming, with volunteers scoring high on this dimension showing greater priming effects than those scoring low. We focus on the cognitive disorganization dimension of schizotypy because it is assumed to be the most closely related 
314 with thought disorder (Mason \& Claridge, 2006; Raine, 1991). We predicted that, on average, 315 semantic priming would be observed as a difference in response latencies such that responses

316 were faster to word targets with related primes than to targets with unrelated primes. However, 317 we predicted an interaction such that this related vs. unrelated difference would be larger for 318 participants with higher scores on cognitive disorganization scales. Although, as we have already 319 mentioned, the disorganized dimension of the SPQ might be more adequate to assess thought 320 disorder than the corresponding dimension of the O-LIFE, we included versions of both scales in 321 our study in order to facilitate comparisons with previous research.

322

323 We aimed to test, secondly, whether the association between schizotypal personality and 324 semantic memory relies on processes that are automatic (i.e. enhanced automatic spreading of 325 semantic activation) or controlled (e.g. attentionally-driven expectancy or matching effects). We 326 hypothesized that if variation between individuals in semantic priming reflected the impact of 327 schizotypy on either automatic or controlled semantic processes then we should expect to 328 observe an interaction between the effects of individual differences in disorganization, stimulus 329 onset asynchrony (SOA), and prime-target relatedness. If observed semantic priming was 330 associated with automatic or with controlled processes, then the priming effect should be 331 modulated by SOA (yielding an SOA by priming interaction). If the expression of schizotypy in 332 priming was related to either automatic or controlled processes then this SOA by priming 333 interaction should, itself, by modulated by individual differences in (cognitive) disorganization 334 (yielding a disorganization by SOA by priming interaction). 335

Finally, we reasoned that if schizotypal personality implies illogical semantic storage or furtherreaching spreading of semantic activation then we should observe priming effects when prime 338 and target were indirectly related. We predicted that, on average, priming would be reflected in faster responses to targets with related than to targets with unrelated primes but that priming would be greater for directly than for indirectly related primes (yielding an interaction between 341 the effects of relatedness and of the directness of the relation). However, we hypothesized that 342 the association between schizotypy and semantic processing should be evident in both indirect 343 and direct priming conditions for individuals scoring high on disorganization. This implies the 344 prediction that the directness $\mathrm{x}$ priming interaction should be smaller (the impact of directness on 
345 priming should be reduced) in individuals scoring higher on disorganization, that is, priming 346 should be more similar under direct and indirect prime-target relatedness conditions for 347 individuals scoring higher on disorganization. In the following, we report an investigation in 348 which we manipulated the directness of prime-target relatedness in two sub-studies (direct, 349 indirect priming).

350

\section{Materials \& Methods}

352

353 Participants

354 A group of 81 Psychology students from the University of Barcelona took part in a direct 355 priming sub-study in exchange for course credits (mean age $=19.8, \mathrm{SD}=1.9 ; 73$ females; 69 356 self-reported as right-handed). A group of 79 different volunteers from the same pool took part in 357 the indirect priming sub-study (mean age $=19.67, \mathrm{SD}=1.75 ; 71$ females; 72 right-handed).

358 They were all native speakers of Spanish with normal or corrected-to-normal vision who 359 reported no history of neurological or psychiatric disease. We gathered written informed consent 360 prior to their participation. The study protocols were approved by the university's ethics 361 committee (1RBOOOO3O99, Comissió de Bioètica de la Universitat de Barcelona, CBUB). All

362 data were recorded anonymously.

363

364

Materials

365 We selected 60 prime-target pairs from the Normas de Asociación Libre en Castellano database, 366 which provides lists of free association data for different words in Spanish. For the direct 367 priming sub-study, we selected a related target for each prime from the two most associated 368 words in its list, avoiding phonological overlap between primes and targets. The unrelated prime369 target pairs were constructed by pseudorandomly distributing the targets to different primes, 370 while avoiding semantic association and phonological overlap among the unrelated prime-target 371 pairings. For each target word, we created an orthographically plausible pseudoword with the 372 software Wuggy (Keuleers \& Brysbaert, 2010). The pseudowords matched the letter length, 373 length of subsyllabic segments and transitional frequencies of the real words.

375 In order to select new targets indirectly associated to the original primes for the indirect priming 376 sub-study, we chose new words from the two most associated words in the directly associated 
377 targets' lists of associates. Stimuli that appeared as directly related words in the corresponding 378 prime's list of associates, or words phonologically similar to them, were not included in the final 379 selection. Although, for clarity, we explain stimuli selection for the two sub-studies separately, 380 we selected directly and indirectly related pairs simultaneously.

381

382 383

384 385 386

387 388

389

390 391

392 393 394 395 396 397 398 399 400 401 402 403 404 405

406 Reliability values corresponding to our participants' responses to this inventory were:

407 disorganized, mean $=4.49, \mathrm{SD}=2.82, \alpha=0.76$; positive, mean $=2.68, \mathrm{SD}=2.1, \alpha=0.63$;

We measured schizotypal personality by means of two questionnaires: the brief versions of the SPQ (SPQ-B, Raine \& Benishaw, 1995) and the O-LIFE (sO-LIFE, Mason et al., 2005). We translated these questionnaires into Spanish following common translation and back-translation procedures (Sierro et al., 2016). First, a native Spanish speaker of advanced English proficiency translated the English versions into Spanish. Then, an English native bilingual professional translator back-translated the Spanish versions. The minor differences revealed by comparing the original and back-translated versions were discussed by the two translators until agreement. The translated scale items are available at OSF (https://osf.io/j29fn/?view_only=6eb7d8dfb7c34e62be21a0ba95f61020).

The SPQ-B consists of 22 items assessing cognitive-perceptual deficits (positive dimension, e.g. "Have you ever had the sense that some person or force is around you, even though you cannot see anyone?"), interpersonal deficits (negative dimension, e.g. "People sometimes find me aloof and distant") and disorganization (disorganized dimension, e.g. "People sometimes comment on my unusual mannerisms and habits"). Following Ferchiou et al. (2017), our participants responded to the SPQ-B questionnaire using a 5-point Likert-like scale with 1 indicating "completely disagree" and 5 indicating "completely agree". Reliability values for responses of our full participant sample $(n=160)$ were: disorganized, mean $=13.53, \mathrm{SD}=4.71, \alpha=0.78$; positive, mean $=17.66, \mathrm{SD}=5.59, \alpha=0.75$; negative, mean $=19.89, \mathrm{SD}=6.65, \alpha=0.84$. The sO-LIFE consists of 43 yes/no questions tapping into positive traits (e.g. "When in the dark do you often see shapes and forms even though there is nothing there?"), negative traits (e.g. "Are there very few things that you have ever enjoyed doing?"), disorganized traits (e.g. "Are you easily confused if too much happens at the same time?") and impulsive non-conformity traits (e.g. "Do you consider yourself to be pretty much an average sort of person?" - reversed). 
408 negative, mean $=2.7, \mathrm{SD}=1.77, \alpha=0.62$; impulsive nonconformity, mean $=2.39, \mathrm{SD}=1.8, \alpha=$

409 0.54. The participants in the direct and indirect priming sub-studies showed significant

410 differences in their cognitive disorganization scores as measured by SPQ-B (direct priming mean

$411=14.5, \mathrm{SD}=4.8$; indirect priming mean $=12.6, \mathrm{SD}=4.4 ; t(158)=2.560, p=.011)$, but not by $\mathrm{SO}-$

412 LIFE.

413

414 Design and procedure

415 We manipulated the directness of the relation between prime and target in two separate sub-

416 studies conducted with different participants. The two studies were identical in procedure but

417 differed in the target stimuli (which were directly or indirectly related to the primes).

418

419 In summary, each participant completed 480 lexical decision trials, completing 240 trials

420 responding to word targets and 240 trials to pseudoword targets. In both studies, SOA and

421 relatedness were manipulated within-primes, -targets and -participants. Each participant saw

422 each prime eight times, four times with word targets and four times with pseudoword targets. For

423 the four trials in which a word target was presented, each participant saw the prime two times

424 with one related target (e.g. abdomen-BARRIGA), once at the short SOA and once at the long

425 SOA, and they saw the prime another two times with one unrelated target (e.g. abdomen-MES),

426 once at each SOA. Each person saw each word target four times, twice under the related

427 condition (e.g. abdomen-BARRIGA), once at the short and once at the long SOA, and twice

428 under the unrelated condition (e.g. abeja-BARRIGA), once at the short and once at the long

429 SOA.

430

431 For each SOA condition (short or long), we presented the stimuli in two blocks of 120 trials

432 each. Stimuli were presented in different SOA conditions in one of two blocks (short vs. long

433 SOA blocks) to reduce the impact of repetition priming. The order of the blocks and the order of

434 the trials within each block were randomized, and the order of administration of the conditions

435 was counterbalanced across participants. The studies started with four practice trials.

436

437 The task was administered using the DMDX software application (Forster \& Forster, 2003).

438 Stimuli were presented in 4:3 CRT screens with $1024 \times 768$ resolution using Arial 14pt font. 
439 Participants were seated $35 \mathrm{~cm}$ from the screen. Each trial started with a "+" presented as fixation 440 point for $500 \mathrm{~ms}$. Then, the prime appeared for $200 \mathrm{~ms}$ followed by the target word after $50 \mathrm{~ms}$ or $441550 \mathrm{~ms}$, respectively, for the short or long SOA conditions. The target was onscreen for $200 \mathrm{~ms}$ 442 and was followed by a blank screen for 2000ms until the next trial began (the response interval) 443 during which participants were able make keypress responses to indicate lexical decisions to 444 targets stimuli (pressing " $\mathrm{M}$ " for real words with their right hand and " $Z$ " for pseudowords with 445 their left hand on a QWERTY-type keyboard). The participants were tested in groups of up to 446 four in clone set-ups.

447

\section{Results}

449 The full dataset and code for the analyses are available at OSF

450 (https://osf.io/j29fn/?view_only=6eb7d8dfb7c34e62be21a0ba95f61020).

451

452 Explanation of analysis approach

453 In our analyses, we used the brms library (Bayesian regression models using 'Stan'; Bürkner, 454 2017, 2019; Carpenter et al., 2017) to fit Bayesian mixed-effects models. A detailed explanation 455 of our analysis approach is set out in Supplementary Materials (see Supplemental Article S1) but 456 we summarize the motivation for our approach here. For each effect, we assumed that coefficient 457 estimates may vary in sign and magnitude. Bayesian models are scientifically advantageous 458 because they yield a posterior probability distribution representing the differing probabilities of 459 each potential value of an effect, given the observed evidence and given prior expectations about 460 likely effects. This means that, for each effect, we are able to report the most probable value of 461 the estimate for the effect, while the spread of the posterior distribution directly indicates our 462 uncertainty about the estimate. We report credible intervals (CrI) to summarize that uncertainty. 463

464 The study design required the manipulation of prime-target relatedness, the directness of the 465 prime-target relation, and prime-target stimulus onset asynchrony, in addition to the observation of participants' scores on the SPQ-B and SO-LIFE measures of variation in schizotypy dimensions. We sum-coded $(-1,+1)$ the effects of the categorical variables: prime-target relatedness; SOA; and directness of prime-target relatedness. We standardized participants' scores on the schizotypy dimensions. We fitted separate models including the effects of 
470 participant variation on each dimension of one set of schizotypy scales (sO-LIFE or SPQ-B)

471 only. Models were structured to estimate effects of these variables as well as the effects of all

472 interactions up to and including the potential four-way interaction between the effects of

473 directness, schizotypy dimension, SOA and relatedness. We fitted models that included

474 parameters corresponding to random effects associated with:

475 (1.) unexplained differences between sampled participants in intercepts (random intercepts) and 476 in the within-participant effects of SOA, relatedness and the SOA x relatedness interaction

477 (random slopes); as well as correlations between random intercepts and random slopes;

478 (2.) unexplained differences between sampled primes or targets in intercepts (random intercepts) 479 and in the within-stimulus effects of participants' variation in schizotypy dimensions, and in the 480 effects of SOA, relatedness and the SOA x relatedness interaction (random slopes); as well as 481 differences in the within-prime effect of directedness; along with correlations between random 482 intercepts and random slopes. (See Meteyard \& Davies, 2020, for a discussion of the 483 specification of fixed and random effects.)

484

485 In the present article, we report the posterior distributions of parameter estimates yielded by 486 models assuming weakly informative priors for fixed effects coefficients or random effects 487 variances: Gaussian (normal) probability distributions centered on a mean of zero with a 488 standard deviation of $10(\beta \sim \operatorname{Normal}(0,10) ; S D \sim \operatorname{Normal}(0,10))$. This assumption of priors 489 expresses the belief that the parameter values would lie between -20 and +20 with $95 \%$ 490 probability. To examine the sensitivity of our results to our assumptions, we fitted a series of 491 models with the same fixed and random effects structures but varying prior probability 492 distributions. These sensitivity analyses (see Supplemental Article S2) indicate that the estimates 493 derived from our models are stable across a range of alternate assumptions. We also conducted 494 frequentist versions of our analyses which showed similar results (see Supplemental Article S3) 495 though the most complex models were associated with convergence problems.

497 Analysis findings

498 We report our findings from, firstly, a model including SPQ-B measures of individual 499 differences on schizotypy dimensions, and, secondly, a model including sO-LIFE measures of 500 differences on schizotypy. 
501

$502 S P Q-B$ model

503 We begin by reporting the SPQ-B model effects estimates, together with a quantification of our

504 uncertainty about those estimates, by presenting visualizations of Markov Chain Monte Carlo

505 (MCMC) draws from the posterior distribution of the parameters of the Bayesian model,

506 produced using the bayesplot library (Gabry, 2017; Gabry et al., 2019). Fig. 1 presents density

507 plots computed from posterior draws with all chains merged, with the uncertainty intervals $(95 \%$

508 intervals) shown as the shaded areas under the curves. The plots indicate, firstly, that the most

509 probable estimates (located at the vertical lines) for many effects is close to zero. For these

510 effects, including the effects of variation in SPQ-B positive, negative or disorganization

511 dimensions, the impact of individual differences in schizotypy on the latency of correct word

512 classifications was very small. In contrast, we found evidence for effects of SOA, relatedness,

513 and the interaction between relatedness and the directness of prime-target relatedness.

514

515 [Figure 1 about here.]

516 Figure 1. SPQ analysis - posterior distribution plot for each effect

517

518 We present a summary of the model in Table 2, showing just the estimates for the effects of the 519 experimental variables and of individual differences on schizotypy dimensions (a full table 520 summary, including fixed and random effects, is presented in Supplemental Table 1). Every

521 effect is summarized using the mean and the standard error of the posterior distribution together 522 with the upper and lower limits of two-sided 95\% credible intervals (lower and upper bounds)

523 based on quantiles. (Model summary tables also include Rhat values, a metric of convergence

524 (Vasishth et al., 2018) which should be (and is) close to 1.)

525

526 [Table 2 about here]

527 Table 2. SPQ model summary of population-level effects estimates

528

529 Response latencies were about 16ms longer under the long compared to the short SOA

530 conditions $(\hat{\beta}=7.76,95 \% \operatorname{CrI}=[4.08,11.42])$. (Note that, given the sum coding for the factor -

531 - short SOA $=-1$, long SOA $=+1$-- contrasts are constrained to sum to zero so that the 
532 coefficient of the effect corresponds to the difference between the mean latency at one condition

533 level, i.e., long SOA, compared to the overall mean across levels, at 0 , halfway between short

534 and long SOA.) Response latencies were about $14 \mathrm{~ms}$ shorter under the related compared to the

535 unrelated priming conditions $(\hat{\beta}=-7.11,95 \% \operatorname{CrI}=[-8.85,-5.35])$, with relatedness also

536 sum coded (unrelated $=-1$, related $=+1$ ). Latencies were about $18 \mathrm{~ms}$ longer in the direct (

537 coded $=1)$ compared to the indirect $(=-1)$ condition $(\hat{\beta}=8.49,95 \% \operatorname{CrI}=[-0.77,17.47])$

538 but the evidence for the effect was relatively weak or uncertain because the breadth of the

539 credible interval encompasses quite broad uncertainty about the magnitude or sign of the effect.

540

541 We observed evidence for an interaction between the relatedness and the directness effects. The

542 (negative) effect of prime relatedness was about $5 \mathrm{~ms}$ greater under the direct compared to the

543 indirect priming conditions, given the effect of the interaction $(\hat{\beta}$

$544=-4.52,95 \% \operatorname{CrI}=[-6.12,-2.92])$. Fig. 2 illustrates the trends in RT differences associated

545 with this interaction. In contrast, there is little evidence for interactions between SOA and

546 relatedness or between SPQDis differences and relatedness. The points in the plot indicate the

547 mean model fitted outcome, given different values of each predictor variable, conditional on all

548 other predictor variables set to their mean values. The error bars indicate the upper and lower

549 bounds of the $95 \%$ credible intervals for the estimates.

550

551 [Figure 2 about here.]

552

Figure 2. SPQ analysis - marginal effects plot for two-way interaction effects

553

554 We hypothesized that individual differences on schizotypy dimensions would modulate: (1.) the 555 effect of relatedness, predicting a disorganization by relatedness interaction; (2.) the effect of the

556

557

558

559

560

561

562 interaction between SOA and relatedness, predicting a disorganization by SOA by relatedness interaction; and (3.) the effect of the interaction between directness and relatedness, predicting a disorganization by directness by relatedness interaction. The posterior distribution plots shown in Fig. 1 clearly indicate how, in contrast to these expectations, the estimates for the effects of the critical interactions were all close to zero.

\section{sO-LIFE model}

Peer) reviewing PDF | (2019:10:42406:2:0:NEW 9 Jun 2020) 
563 The model including sO-LIFE instead of SPQ-B schizotypy dimensions presents a largely

564 similar pattern of effects. For this reason, we present just the tabled summaries of the posterior

565 distribution for parameters (see Table 3), and we invite interested readers to inspect the posterior

566 distribution and marginal effects plots in Supplemental Materials (Fig. S1 and S2). (A full table

567 summary, including fixed and random effects, is presented in Supplemental Table 2.)

568

569 [Table 3, about here]

570 Table 3. OLIFE model summary of population-level effects estimates

571

572 What is distinct, compared to the SPQ model, are the indications, firstly, that for at least one

573 schizotypy dimension, OLIFEImp, the most probable effect of variation on that dimension is that

574 response latencies changed perceptibly - RTs increased - albeit by a small amount, in

575 association with increasing scores on the measure $(\hat{\beta}=5.56,95 \% \operatorname{CrI}=[-4.30,15.29])$. The

576 plot presenting the posterior distribution for the effect of OLIFEImp indicates that the estimated

577 effect coefficient is relatively small (under 10ms), and the uncertainty about the nature of the

578 effect is quite broad, suggesting weak evidence that is perhaps insufficient to resolve the nature

579 of the trend.

580

581 The sO-LIFE model suggests, in addition, that individual differences on some schizotypy

582 dimensions did appear to modulate the effects of the experimental variables but that that

583 influence was observed as subtle, rather small, modulations. Thus, we observed some evidence

584 for potentially interesting interactions. We see some weak evidence for an interaction between

585 the effects of variation in OLIFEDis scores and the impact of directness condition $(\hat{\beta}$

$586=4.52,95 \% \operatorname{CrI}=[-5.20,14.22])$, indicating that for individuals with higher OLIFEDis

587 scores the difference between RTs under direct compared to indirect priming conditions tended

588 to increase. We also see some evidence for an interaction between the effects of variation in

589 OLIFEImp scores and of variation in $\operatorname{SOA}(\hat{\beta}=5.00,95 \% \mathrm{CrI}=[0.91,9.12])$, indicating that

590 for individuals with higher OLIFEImp scores the difference between RTs under long compared

591 to short SOA conditions tended to increase. Lastly, the model indicated some evidence for a

592 small effect associated with the three-way interaction between the effects of the directness of

593 relation, of variation in OLIFEImp scores, and of relatedness $(\hat{\beta}=2.12,95 \% \operatorname{CrI}=[0.47,3.76]$. 
594 However, the effect is so small that it can be said to have corresponded to a negligible influence 595 on performance in the lexical decision task.

596

597 Discussion

598 Our study aimed to ascertain whether individual differences in schizotypal personality, 599 specifically, disorganized traits, are associated with increased spreading of semantic activation.

600 This possibility is of substantive theoretical and practical importance because it could explain 601 symptoms, such as thought disorder, in clinical or sub-clinical populations. Although this

602 hypothesis has been present in the literature for years now, results that have been adduced to 603 support it are far from definitive so that it has not been clear under what circumstances, and in 604 what shape, the association should be observed.

605

606 Regarding the impact of a direct semantic relation between lexical stimuli in word recognition 607 tasks, evidence from two previous studies have indicated a significant association between 608 variation in schizotypy and increased priming effects, either under automatic (short SOA) 609 priming conditions (Neill et al., 2014) or under both automatic and controlled priming conditions 610 (Moritz et al., 1999). This has led to the claim that individuals scoring high on schizotypy 611 present increased spreading of semantic activation (Neill et al., 2014) compared to individuals 612 scoring low on schizotypy. With respect to evidence of the priming that may result from 613 indirectly related prime-target lexical stimulus pairs, the observation of increased priming effects 614 among individuals scoring high on schizotypy, under both automatic (Johnston et al., 2008;

615 Moritz et al., 1999) and controlled priming conditions (Rossell et al., 2014) has led to the 616 conclusion that individuals with schizotypal traits might present further-reaching spreading of 617 semantic activation (Johnston et al., 2008).

618

619 Our study does not support any of these hypotheses. We tested a sample of participants ( $\mathrm{n}=160$, 620 in total), with a sample of lexical stimuli (240 word trials per person), considerably larger than 621 that employed in most studies reported in this area. Moreover, we overcame the limitations of 622 previous work by conducting Bayesian mixed-effects models to estimate the effects of the 623 experimental variables (prime-target relatedness, stimulus onset asynchrony (SOA), the 624 directness of the prime-target relation) as well as the effects of individual differences on 
625 schizotypal dimensions, and, critically, the effects of interactions between the impact of the 626 experimental conditions and variation on schizotypy. This approach yielded a high level of 627 sensitivity in our analyses: sufficient for us to be able to identify, with a high degree of certainty, 628 small but distinct effects due to the relatedness of prime-target pairs (a semantic priming effect), 629 the length of SOA, and the modulation of the relatedness effect by the directness of the relation 630 between primes and targets. Indeed, our analyses were sufficient to pick up subtle deviations 631 among participants (estimated as random effects variances) in average reaction time, or in the 632 effects of prime-target relation, of SOA, and of the interaction between relatedness and SOA (see 633 Supplemental Tables S1 and S2). Impressively, our analyses were sufficient to pick up random 634 variation among responses due to differences between primes in the slopes of the effects of 635 relatedness, directness, SOA or schizotypy variation. These random effect variances were 636 relatively small (SDs of the order of $<5$ ) but within the capacity of our methods to derive 637 precise estimates. Yet we observed no influence of cognitive disorganization, and no interaction 638 between the effects of cognitive disorganization and semantic relation.

639

640 We acknowledge that it is possible that our participant sample was not sufficient, or not 641 sufficiently representative of the distribution of individual differences in cognitive 642 disorganization in the wider population. However, a comparison of the distribution of our 643 participants' scores with those obtained in previous studies indicate that our sample can be 644 considered to be representative of samples tested in research in the field, potentially, therefore, of 645 the distribution in the general population. Regarding scores in sO-LIFE, the results of our 646 participants covered the full range of possible scores and their distribution characteristics 647 resemble those of the sample assessed in the original scaling study (cognitive disorganization 648 scores in our study: mean $=4.49, \mathrm{SD}=2.82$; in Mason et al. $(2005)$ : mean $=4.42, \mathrm{SD}=2.9)$. 649 Regarding scores in the SPQ-B, our results showed slightly lower scores but greater variability 650 than those obtained in scaling studies conducted with an analogous Likert-based version of the 651 test (disorganization scores in our study: mean =13.53, $\mathrm{SD}=4.71$; in Ferchiou et al. (2017): 652 mean $=16.77, \mathrm{SD}=1.13$ ). In relation to this, future studies might be interested in assessing a 653 sample comprising both subclinical individuals as well as patients in order to cover the full 654 continuum of thought disorder prevalence. 
656 It should be noted that our analyses indicated a small difference in cognitive disorganization

657 scores as measured by SPQ-B (but not by sO-LIFE) between participants in the direct and

658 indirect priming sub-studies. This difference could be argued to potentially limit the

659 interpretability of the directness effect observed in our research. However, we believe that if a

660 between-group difference in disorganization was confounded with the between-group difference

661 in type of priming (direct vs. indirect) then we should expect the directness effect to be observed

662 in the SPQ-B analysis but not in the sO-LIFE analysis. Instead, we observed some evidence for a

663 directness effect in both analyses though we note that the credible interval suggested

664 considerable uncertainty over the magnitude of the effect.

665

666 Our study focused on examining the possible association between variability in semantic

667 processing and differences in thought disorder as measured by cognitive disorganization scales

668 of schizotypy questionnaires in sub-clinical volunteers. However, some authors have suggested

669 that disturbances in semantic processing could also be associated with individual differences in

670 positive traits such as dissociative experiences (Dehon et al., 2008; Winogard et al., 1998)

671 hallucinations (Kanemoto et al., 2013; Sugimori et al., 2011) or paranormal beliefs (Meyersburg

672 et al., 2009; Pizzagalli et al., 2001). Our study is also informative in relation to this hypothesis

673 because we employed the questionnaire measures (SPQ-B and sO-LIFE) to estimate individual

674 differences not only in disorganized traits, but also in positive and negative traits. Mirroring the

675 results obtained in relation to disorganized traits, our analyses indicated little or no evidence for

676 interactions between differences in positive symptoms and semantic priming effects.

677

678 At least two questions regarding the representativeness of our sample deserve attention. Firstly, 679 around $90 \%$ of our participants were females in both sub-studies. Differences in the distribution 680 of schizotypal traits between males and females have been reported in previous work. For 681 instance, Mason and Claridge (2006) reported significantly higher cognitive disorganization

682 scores for males compared to females during the creation of O-LIFE. Given the gender

683 imbalance in our sample, our evidence cannot be used to examine whether the interplay between

684 schizotypal traits and semantic processing is affected by gender. Further studies with comparable

685 male and female samples should be conducted to test this possibility. 
687 Another relevant issue concerns the handedness of our participants. Although most self-

688 identified as right-handed, we did not conduct any systematic assessment of their degree of hand

689 preference. Ambilaterality has been associated with schizotypal traits such as magical thinking

690 (Barnett \& Corballis, 2002), and functional hemispheric asymmetry, which is known to be 691 associated with handedness (Bourne, 2006), has been suggested to share a neural basis with 692 schizotypy (Schmitz et al., 2019). Along these lines, previous studies have reported stronger 693 priming effects in paranormal believers, compared to disbelievers, only after left visual field 694 presentation of the words (Mohr et al., 2006; Pizzagalli et al., 2001). Taking these observations 695 into account, recruitment of homogeneous right-handed samples or samples including comparable groups of right-handed and left-handed participants and left-lateralized presentation

697 of priming stimuli might be more adequate to capture the relation between schizotypal traits and 698 semantic processing and should be considered in further studies.

699

700

In our view, the findings we report suggest that previous observations regarding the association

701 between cognitive disorganization and priming should be revisited. As we have already outlined

702 in the introduction, the results in this area present some inconsistency. Yet the potential

703 implications of answers to the research question, whether variation in schizotypy influences the degree or extent of semantic activation, seem to us to merit further research in this area. Our findings indicate that that further research will require the completion of studies that are sufficiently powered to permit the identification of what are likely to be subtle interactions.

707

708 One limitation of our study is related to the use of self-report schizotypal questionnaires to 709 measure cognitive disorganization. These kinds of assessment tools have been predominant in the literature studying the relation between schizotypy and semantic priming effects. In our work, we used the short versions of the two questionnaires most commonly used in previous

712 studies, focusing on the disorganization dimensions. In the SPQ-B, this dimension includes items 713 tapping into odd speech (directly related to thought disorder) and odd behavior. Regarding sO-

714 LIFE, the analogous dimension of cognitive disorganization includes items specifically assessing

715 thought disorder, but also other traits such as poor attention and decision-making or social 716 anxiety. Taking this into account, the cognitive disorganization scales of these two 717 questionnaires might not be specific enough to precisely assess thought disorder, not to mention, 
718 to capture the usually overlooked heterogeneity of this phenomenon (Sass \& Parnas, 2017). In

719 this sense, more objective measures of this trait, such as word graph analyses (Mota et al., 2012,

720 2017) or repetition and predictability indexes (Linscott, 2005) might be more adequate to capture

721 the possible association between schizotypy and variability in semantic processing in future

722 studies.

723

\section{Conclusions}

725 In sum, our study provides no compelling evidence that schizotypal traits, specifically those 726 associated with the cognitive disorganization dimension, reflect enhanced semantic processing,

727

728

729

730

731

732

733

734

735

736

737

738

739

740

741

742

743

744

745

746

747

as evidenced by variation in semantic activation in priming tasks. Our findings warrant the conclusion that the effects of interest may be relatively small, and thus require high powered investigations for precise estimation in future work.

\section{Acknowledgements}

All analyses were run using The High End Computing (HEC) facility at Lancaster University. We gratefully acknowledge the support of Mike Pacey in using the HEC to complete our analyses.

\section{References}

American Psychiatric Association. (1987). DSM-III-R: Diagnostic and Statistical Manual of Mental Disorders. (3rd ed., r). The Association.

Barnett, K. J., \& Corballis, M. C. (2002). Ambidexterity and magical ideation. Laterality, 7(1), 75-84. https://doi.org/10.1080/13576500143000131

Barrantes-Vidal, N. (2004). Creativity \& madness revisited from current psychological perspectives. Journal of Consciousness Studies, 11(3-4), 58-78.

Bourne, V. J. (2006). The divided visual field paradigm: Methodological considerations. Laterality, 11(4), 373-393. https://doi.org/10.1080/13576500600633982

Bürkner, P. (2017). brms: An R Package for Bayesian Multilevel Models Using Stan. Journal of Statistical Software, 80, 1-28.

Bürkner, P. (2019). brms: Bayesian Regression Models using “Stan” (R package version 
748

749

750

751

752

753

754

755

756

757

758

759

760

761

762

763

764

765

766

767

768

769

770

771

772

773

774

775

776

777

778

2.10.0.). https://cran.r-project.org/web/packages/brms/brms.pdf

Callaway, E., \& Naghdi, S. (1982). An Information Processing Model for Schizophrenia. Archives of General Psychiatry, 39(3), 339-347. https://doi.org/10.1001/archpsyc.1982.04290030069012

Carpenter, B., Gelman, A., Hoffman, M., Lee, D., Goodrich, B., Betancourt, M., Brubaker, M. A., Guo, J., Li, P., \& Ridell, A. (2017). Stan: A Probabilistic Programming Language. Journal of Statistical Software, 76, 1-32.

Cohen, J. (1983). The cost of dichotomization. Applied Psychological Measurement, 7, 249-253.

Cohen, J., Cohen, P., West, S. G., \& Aiken, L. S. (2003). Applied Multiple Regression/Correlation Analysis for the Behavioral Sciences. Lawrence Earlbaum Associates.

Collins, A. M., \& Loftus, E. F. (1975). A spreading-activation theory of semantic processing. Psychological Review, 82(6), 407-428.

Corlett, P. R., Simons, J. S., Pigott, J. S., Gardner, J. M., Murray, G. K., Krystal, J. H., \& Fletcher, P. C. (2009). Illusions and delusions: relating experimentally-induced false memories to anomalous experiences and ideas. Frontiers in Behavioral Neuroscience, $3(53), 1-9$.

Dehon, H., Bastin, C., \& Larøi, F. (2008). The influence of delusional ideation and dissociative experiences on the resistance to false memories in normal healthy subjects. Personality and Individual Differences, 45, 62-67.

Doughty, O. J., \& Done, D. J. (2009). Is semantic memory impaired in schizophrenia? A systematic review and meta-analysis of 91 studies. Cognitive Neuropsychiatry, 14, 473509.

Ettinger, U., Meyhöfer, I., Steffens, M., Wagner, M., \& Koutsouleris, N. (2014). Genetics, cognition, and neurobiology of schizotypal personality: a review of the overlap with schizophrenia. Frontiers in Psychiatry, 5(18), 1-16.

Ferchiou, A., Todorov, L., Lajnef, M., Baudin, G., Pignon, B., Richard, J.-R., Leboyer, M., Szöke, A., \& Schürhoff, F. (2017). Analyse de la structure factorielle de la version brève du questionnaire de personnalité schizotypique (SPQ-B) - format Likert - en population générale en France. L'Encéphale, 43(6), 558-563.

https://doi.org/10.1016/J.ENCEP.2016.05.011

Peer) reviewing PDF | (2019:10:42406:2:0:NEW 9 Jun 2020) 
779 Forster, K. I., \& Forster, J. C. (2003). DMDX: A Windows display program with millisecond

780

781

782

783

784

785

786

787

788

789

790

791

792

793

794

795

796

797

798

799

800

801

802

803

804

805

806

807

808

809 accuracy. Behavior Research Methods, Instruments and Computers, 35, 116-124.

Gabry, J. (2017). bayesplot: plotting for Bayesian models (R Package Version 1.3.0.). http://mcstan.org/bayesplot

Gabry, J., Simpson, D., Vehtari, A., Betancourt, M., \& Gelman, A. (2019). Visualization in Bayesian workflow. Journal of the Royal Statistical Society: Series A (Statistics in Society), $182,389-402$.

Gelman, A., \& Carlin, J. (2014). Beyond power calculations: Assessing Type S (sign) and Type M (magnitude) errors. Perspectives on Psychological Science, 9, 641-651.

Gelman, A., \& Hill, J. (2007). Data analysis using regression and hierarchical/multilevel models. Cambridge University Press.

Hasenfus, N., \& Magaro, P. (1976). Creativity and schizophrenia: an equality of empirical constructs. In British Journal of Psychiatry (Vol. 129, Issue 10, pp. 346-349). https://doi.org/10.1192/bjp.129.4.346

Hori, H., Ozeki, Y., Terada, S., \& Kunugi, H. (2008). Functional near-infrared spectroscopy reveals altered hemispheric laterality in relation to schizotypy during verbal fluency task. Progress in Neuro-Psychopharmacology \& Biological Psychiatry, 32, 1944-1951.

Howell, D. C. (2016). Fundamental statistics for the behavioral sciences. Nelson Education. Johnston, A. E., Rossell, S. L., \& Gleeson, J. F. (2008). Evidence of semantic processing abnormalities in schizotypy using an indirect semantic priming task. The Journal of Nervous and Mental Disease, 196, 694-701.

Kanemoto, M., Asai, T., Sugimori, E., \& Tanno, Y. (2013). External misattribution of internal thoughts and proneness to auditory hallucinations: the effect of emotional valence in the Deese-Roediger-McDermott paradigm. Frontiers in Human Neuroscience, 7(351), 1-11.

Keuleers, E., \& Brysbaert, M. (2010). Wuggy: A multilingual pseudoword generator. Behavior Research Methods, 42(3), 627-633. https://doi.org/10.3758/BRM.42.3.627

Kiang, M., \& Kutas, M. (2006). Abnormal typicality of responses on a category fluency task in schizotypy. Psychiatry Research, 145, 119-126.

Kiang, M., Prugh, J., \& Kutas, M. (2010). An event-related brain potential study of schizotypal personality and associative semantic processing. International Journal of Psychophysiology, 75(2), 119-126. http://linkinghub.elsevier.com/retrieve/pii/S0167876009002566 
810 Kliegl, R., Masson, M. E., \& Richter, E. M. (2010). A linear mixed model analysis of masked 811 repetition priming. Visual Cognition, 18, 655-681.

812 Kwapil, T. R., \& Barrantes-Vidal, N. (2015). Schizotypy: looking back and moving forward. 813 Schizophrenia Bulletin, 41, S366-S373.

814 Laws, K. R., \& Bhatt, R. (2005). False memories and delusional ideation in normal healthy 815 subjects. Personality and Individual Differences, 39, 775-781.

816 Linscott, R. J. (2005). Thought disorder, pragmatic language impairment, and generalized 817 cognitive decline in schizophrenia. Schizophrenia Research, 75(2-3), 225-232.

818 https://doi.org/10.1016/j.schres.2004.10.007

819 Lucas, M. (2000). Semantic priming without association: A meta-analytic review. Psychonomic 820 Bulletin \& Review, 7(4), 618-630. https://doi.org/10.3758/BF03212999

821 Mason, O., \& Claridge, G. (2006). The Oxford-Liverpool Inventory of Feelings and Experiences 822 (O-LIFE): further description and extended norms. Schizoprhenia Bulletin, 82, 203-211.

823 Mason, O., Linney, Y., \& Claridge, G. (2005). Short scales for measuring schizotypy.

824 Schizophrenia Research, 78(2-3), 293-296. https://doi.org/10.1016/J.SCHRES.2005.06.020 825 Meehl, P. E. (1962). Schizotaxia, schizotypy, schizophrenia. American Psychologist, 17, 827826838.

827 Meyer, D. E., \& Schvaneveldt, R. W. (1971). Facilitation in recognizing pairs of words:

828 Evidence of a dependence between retrieval operations. Journal of Experimental 829 Psychology, 90(2), 227-234. https://doi.org/10.1037/h0031564

830 Meyersburg, C. A., Bogdan, R., Gallo, D. A., \& McNally, R. J. (2009). False memory propensity 831 in people reporting recovered memories of past lives. Journal of Abnormal Psychology, $832 \quad 118(2), 399-404$. https://doi.org/10.1037/a0015371

833 Minor, K. S., \& Cohen, A. S. (2012). The role of atypical semantic activation and stress in odd 834 speech: Implications for individuals with psychometrically defined schizotypy. Journal of 835 Psychiatric Research, 46(9), 1231-1236.

836 https://doi.org/10.1016/J.JPSYCHIRES.2012.06.001

837 Mohr, C., \& Claridge, G. (2015). Schizotypy - Do not worry, it is not all worrysome.

838 Schizophrenia Bulletin, 41, S436-S443.

839 Mohr, C., Graves, R. E., Gianotti, L. R., Pizzagalli, D., \& Brugger, P. (2001). Loose but normal: 840 a semantic association study. Journal of Psycholinguistic Research, 30(5), 475-483. 
841

842

843

844

845

846

847

848

849

850

851

852

853

854

855

856

857

858

859

860

861

862

863

864

865

866

867

868

869

870

871

http://www.ncbi.nlm.nih.gov/pubmed/11529423

Mohr, C., Landis, T., \& Brugger, P. (2006). Lateralized semantic priming: modulation by levodopa, semantic distance, and participants' magical beliefs. Neuropsychiatric Disease and Treatment, 2(1), 71-84. http://www.ncbi.nlm.nih.gov/pubmed/19412448

Morgan, C., Bedford, N., \& Rossell, S. L. (2006). Evidence of semantic disorganisation using semantic priming in individuals with high schizotypy. Schizophrenia Research, 84, $272-$ 280 .

Moritz, S., Andresen, B., Domin, F., Martin, T., Probsthein, E., Kretschmer, G., Krausz, M., Naber, D., \& Spitzer, M. (1999). Increased automatic spreading activation in healthy subjects with elevated scores in a scale assessing schizophrenic language disturbances. Psychological Medicine, 29(1), 161-170.

Mota, N. B., Copelli, M., \& Ribeiro, S. (2017). Thought disorder measured as random speech structure classifies negative symptoms and schizophrenia diagnosis 6 months in advance. Npj Schizophrenia, 3(1), 1-10. https://doi.org/10.1038/s41537-017-0019-3

Mota, N. B., Vasconcelos, N. A. P., Lemos, N., Pieretti, A. C., Kinouchi, O., Cecchi, G. A., Copelli, M., \& Ribeiro, S. (2012). Speech graphs provide a quantitative measure of thought disorder in psychosis. PLoS ONE, 7(4), e34928. https://doi.org/10.1371/journal.pone.0034928

Neely, J. H., \& Keefe, D. E. (1989). Semantic context effects of visual word processing: A hybrid prospective-retrospective processing theory. In G. H. Bower (Ed.), The psychology of learning and motivation: Advances in research and theory (Vol. 24, pp. 207-248). Academy Press.

Neill, E., Rossell, S. L., \& Kordzadze, M. (2014). Investigating word associations in a schizotypy sample: Contrasting implicit and explicit processing. Cognitive Neuropsychiatry, 19(2), 134-148. https://doi.org/10.1080/13546805.2013.807727

Pizzagalli, D., Lehmann, D., \& Brugger, P. (2001). Lateralized direct and indirect semantic priming effects in subjects with paranormal experiences and beliefs. Psychopathology, 34(2), 75-80. https://doi.org/10.1159/000049284

Pomarol-Clotet, E., Oh, T. M. S. S. S. S., Laws, K. R., \& McKenna, P. J. (2008). Semantic priming in schizophrenia: systematic review and meta-analysis. The British Journal of Psychiatry, 192(2), 1-6. https://doi.org/10.1192/bjp.bp.106.032102 
872 Raine, A. (1991). The SPQ: a scale for the assessment of schizotypal personality based on DSM873 III-R criteria. Schizophrenia Bulletin, 17(4), 555-564.

874 http://www.ncbi.nlm.nih.gov/pubmed/1805349

875 Raine, A., \& Benishaw, D. (1995). The SPQ-B: a brief screening instrument for schizotypal 876 personality disorder. Journal of Personality Disorders, 9(4), 346-355.

877 Rodríguez-Ferreiro, J., \& Aguilera, M. (2019). Schizotypal personality and semantic functioning: 878 Revisiting category fluency effects in a subclinical sample. Psychiatry Research, 271, 365879 369. https://doi.org/10.1016/J.PSYCHRES.2018.11.074

880 Rodríguez-Ferreiro, J., Aguilera, M., \& Davies, R. (2020). Positive schizotypy increases the 881 acceptance of unpresented materials in false memory tasks in non-clinical individuals.

$882 \quad$ Frontiers in Psychology, 11(262). https://doi.org/10.3389/fpsyg.2020.00262

883 Rossell, S. L., Chong, D., O’Connor, D. A., \& Gleeson, J. F. (2014). An investigation of gender 884 differences in semantic processing in individuals with high schizotypy. Psychiatry 885 Research, 215(2), 491-493. https://doi.org/10.1016/j.psychres.2013.11.015

886 Rossell, S. L., Shapleske, J., \& David, A. S. (2000). Direct and indirect semantic priming with 887 neutral and emotional words in schizophrenia: Relationship to delusions. Cognitive 888 Neuropsychiatry, 5(4), 271-292. https://doi.org/10.1080/13546800050199720

889 Sass, L., \& Parnas, J. (2017). Thought disorder, subjectivity, and the self. Schizophrenia Bulletin, 890 43(3), 497-502. https://doi.org/10.1093/schbul/sbx032

891 Saunders, J., Randell, J., \& Reed, P. (2012). Recall of false memories in individuals scoring high 892 in schizotypy: Memory distortions are scale specific. Journal of Behavior Therapy and 893 Experimental Psychiatry, 43, 711-715.

894 Schmitz, J., Fraenz, C., Schlüter, C., Friedrich, P., Kumsta, R., Moser, D., Güntürkün, O., Genç, 895 E., \& Ocklenburg, S. (2019). Schizotypy and altered hemispheric asymmetries: The role of $896 \quad$ cilia genes. Psychiatry Research - Neuroimaging, 294, 110991.

897 https://doi.org/10.1016/j.pscychresns.2019.110991

898 Sierro, G., Rossier, J., Mason, O. J., \& Mohr, C. (2016). French validation of the O-LIFE short 899 questionnaire. European Journal of Psychological Assessment, 32(3), 195-203.

$900 \quad$ https://doi.org/10.1027/1015-5759/a000249

901 Spitzer, M. (1997). A cognitive neuroscience view of schizophrenic thought disorder. 902 Schizophrenia Bulletin, 23, 29-50. 
903 Sugimori, E., Asai, T., \& Tanno, Y. (2011). Sense of agency over thought: External

904 misattribution of thought in a memory task and proneness to auditory hallucination.

905 Conciousness and Cognition, 20, 688-695.

906 Süllwold, L. (1991). Manual zum Frankfurter Beschwerdefragebogen. Springer.

907 Tan, E. J., \& Rossell, S. L. (2017). Disorganised schizotypy is selectively associated with poorer

908 semantic processing in non-clinical individuals. Psychiatry Research, 256, 249-252.

909 https://doi.org/10.1016/J.PSYCHRES.2017.06.067

910 Vasishth, S., Nicenboim, B., Beckman, M. E., Li, F., \& Kong, E. J. (2018). Bayesian data

911 analysis in the phonetic sciences: A tutorial introduction. Journal of Phonetics, 71, 147-

912161.

913 Vul, E., Harris, C., Winkielman, P., \& Pashler, H. (2009). Puzzlingly high correlations in fMRI

914 studies of emotion, personality, and social cognition. Perspectives on Psychological

$915 \quad$ Science, 4(274-290).

916 Winogard, E., Peluso, J. P., \& Glover, T. A. (1998). Individual differences in susceptibility to

917 memory illusions. Applied Cognitive Psychology, 12, S5-S27.

918 Yap, M. J., Hutchinson, K. A., \& Tan, L. C. (2017). Individual differences in semantic priming 919 performance: Insights from the semantic priming project. In M. N. Jones (Ed.), Frontiers of 920 cognitive psychology. Big data in cognitive science (pp. 203-226). Routledge/Taylor \&

921 Francis Group. https://psycnet.apa.org/record/2016-19548-009

922

923 
Figure 1

SPQ analysis - posterior distribution plot for each effect 
Posterior distributions

with means and $95 \%$ intervals

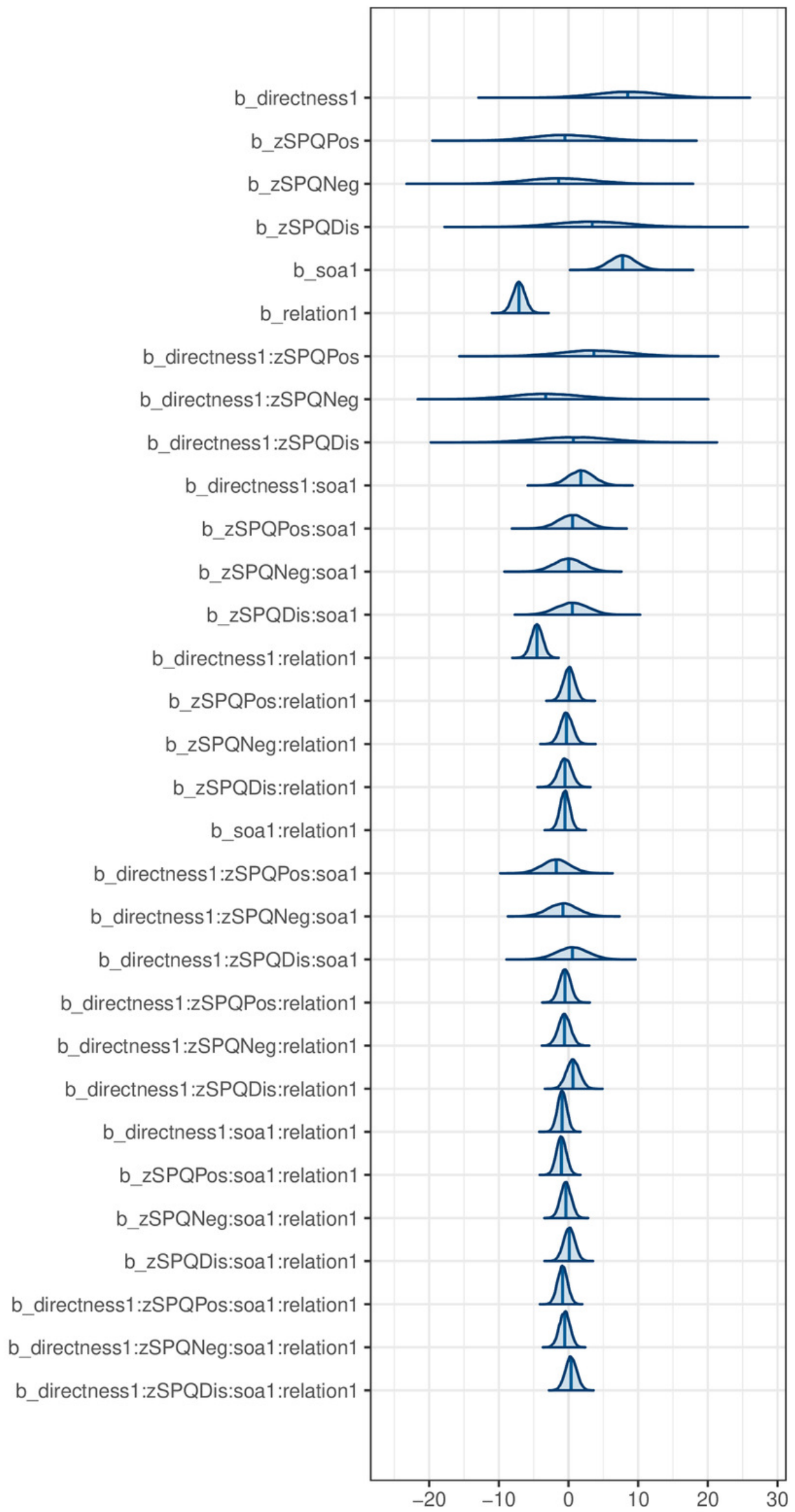


Figure 2

SPQ analysis - marginal effects plot for the two-way interaction effect

A) Directness x relatedness interaction, B) SOA x relatedness interaction, C) Cognitive disorganization $\mathrm{x}$ relatedness interaction
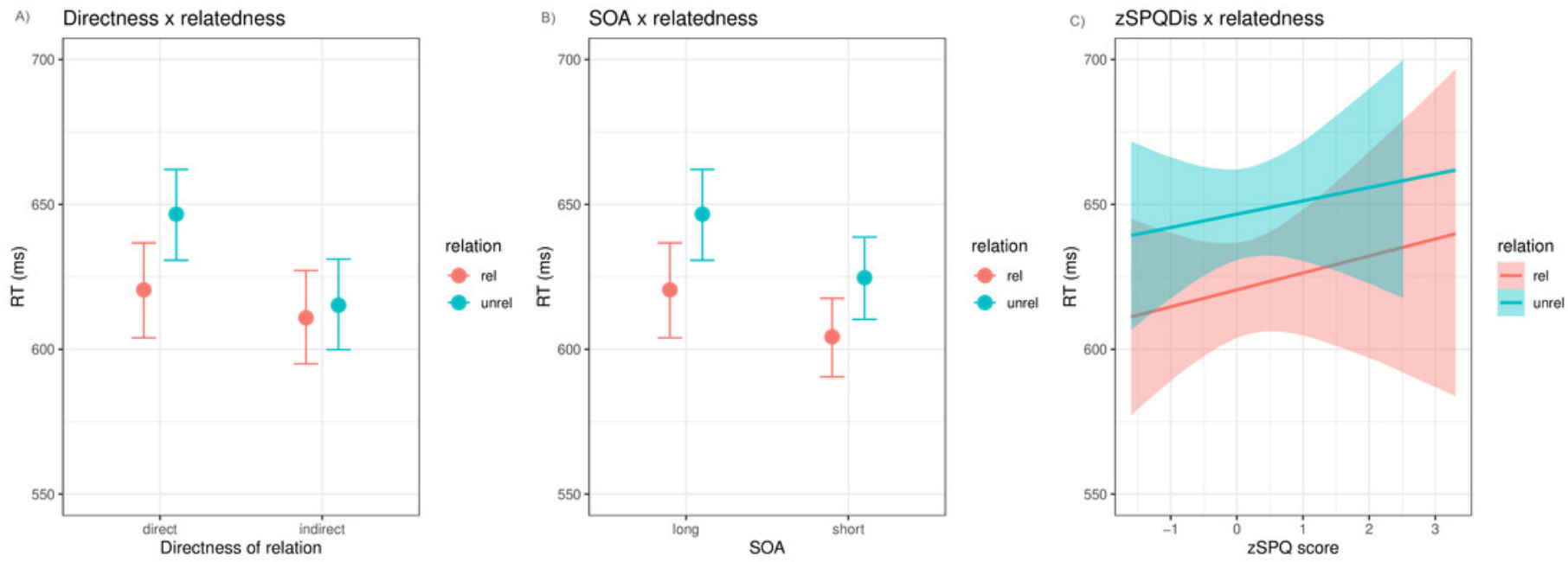


\section{Table $\mathbf{1}$ (on next page)}

Summary of previous studies

SPQ: Schizotypal Personality Questionnaire (Raine, 1991); O-LIFE: Oxford Life Inventory of Feelings and Experiences (Mason \& Claridge, 2006); O-LIFEDis: disorganized subscale of the O-LIFE; Frankfurt: language subscale of the Frankfurt Complaint Questionnaire (Süllwold, 1991); positive: higher scores showed greater priming effects than lower scorers; negative: higher scorers showed smaller priming effects than lower scorers; null: no significant differences between higher and lower scorers or correlation between schizotypy and priming effect; NI: the condition was not included in the study; NA: reaction time data is not available for the study 


\begin{tabular}{|c|c|c|c|c|c|c|c|}
\hline \multirow[b]{2}{*}{ Study } & \multirow[b]{2}{*}{ Questionnaire } & \multirow[b]{2}{*}{$\mathrm{n}$} & \multirow[b]{2}{*}{ Comparison } & \multicolumn{2}{|c|}{ Direct Relation } & \multicolumn{2}{|c|}{ Indirect Relation } \\
\hline & & & & $\begin{array}{l}\text { Short } \\
\text { SOA }\end{array}$ & $\begin{array}{l}\text { Long } \\
\text { SOA }\end{array}$ & $\begin{array}{l}\text { Short } \\
\text { SOA }\end{array}$ & $\begin{array}{l}\text { Long } \\
\text { SOA }\end{array}$ \\
\hline $\begin{array}{l}\text { Moritz et al. } \\
\text { (1999) }\end{array}$ & $\begin{array}{l}\text { SPQ } \\
\text { Frankfurt }\end{array}$ & $\begin{array}{l}156 \\
156\end{array}$ & $\begin{array}{l}\text { median split } \\
\text { median split }\end{array}$ & $\begin{array}{c}\text { null } \\
\text { positive }\end{array}$ & $\begin{array}{c}\text { null } \\
\text { positive }\end{array}$ & $\begin{array}{c}\text { null } \\
\text { positive }\end{array}$ & $\begin{array}{l}\text { null } \\
\text { null }\end{array}$ \\
\hline $\begin{array}{l}\text { Morgan et al. } \\
\text { (2006) } \\
\text { Johnston et }\end{array}$ & O-LIFE & 58 & high vs. low deciles & null & null & $\mathrm{NI}$ & $\mathrm{NI}$ \\
\hline $\begin{array}{l}\text { al. (2008) } \\
\text { Kiang et al. }\end{array}$ & O-LIFEDis & 54 & correlational & null & null & positive & null \\
\hline $\begin{array}{l}\text { (2010) } \\
\text { Neil et al. }\end{array}$ & SPQ & 28 & correlational & NA & NA & NA & NA \\
\hline (2004) & O-LIFEDis & 36 & high vs. low thirds & positive & $\mathrm{NI}$ & negative & $\mathrm{NI}$ \\
\hline $\begin{array}{l}\text { Rossell et al. } \\
\text { (2014) } \\
\text { Tan and }\end{array}$ & O-LIFEDis & 82 & correlational & null & null & null & positive \\
\hline Rossell (2017) & O-LIFEDis & 60 & correlational & null & null & $\mathrm{NI}$ & $\mathrm{NI}$ \\
\hline
\end{tabular}

1 SPQ: Schizotypal Personality Questionnaire (Raine, 1991); O-LIFE: Oxford Life Inventory of Feelings and

2 Experiences (Mason \& Claridge, 2006); O-LIFEDis: disorganized subscale of the O-LIFE; Frankfurt:

3 language subscale of the Frankfurt Complaint Questionnaire (Süllwold, 1991); positive: higher scores

4 showed greater priming effects than lower scorers; negative: higher scorers showed smaller priming

5 effects than lower scorers; null: no significant differences between higher and lower scorers or

6 correlation between schizotypy and priming effect; NI: the condition was not included in the study; NA:

7 reaction time data is not available for the study 
Table 2 (on next page)

$S P Q$ model summary of population-level effects estimates 


\begin{tabular}{|c|c|c|c|c|c|}
\hline Parameter & mean & SE & $\begin{array}{l}\text { lower } \\
\text { bound }\end{array}$ & $\begin{array}{l}\text { upper } \\
\text { bound }\end{array}$ & Rhat \\
\hline Intercept & 615.54 & 5.20 & 605.39 & 625.91 & 1.00 \\
\hline directness & 8.49 & 4.66 & -0.77 & 17.47 & 1.00 \\
\hline zSPQPos & -0.52 & 4.72 & -9.74 & 8.76 & 1.00 \\
\hline zSPQNeg & -1.41 & 4.95 & -11.23 & 8.16 & 1.00 \\
\hline zSPQDis & 3.41 & 5.16 & -6.60 & 13.60 & 1.00 \\
\hline soa & 7.76 & 1.87 & 4.08 & 11.42 & 1.00 \\
\hline relation & -7.11 & 0.89 & -8.85 & -5.35 & 1.00 \\
\hline directness:zSPQPos & 3.64 & 4.81 & -5.85 & 13.01 & 1.00 \\
\hline directness:zSPQNeg & -3.26 & 5.06 & -13.34 & 6.58 & 1.00 \\
\hline directness:zSPQDis & 0.69 & 5.23 & -9.53 & 10.88 & 1.00 \\
\hline directness:soa & 1.78 & 1.84 & -1.88 & 5.37 & 1.00 \\
\hline zSPQPos:soa & 0.58 & 2.02 & -3.36 & 4.59 & 1.00 \\
\hline zSPQNeg:soa & 0.04 & 2.11 & -4.12 & 4.20 & 1.00 \\
\hline zSPQDis:soa & 0.56 & 2.27 & -3.94 & 4.96 & 1.00 \\
\hline directness:relation & -4.52 & 0.82 & -6.12 & -2.92 & 1.00 \\
\hline zSPQPos:relation & 0.09 & 0.82 & -1.54 & 1.69 & 1.00 \\
\hline zSPQNeg:relation & -0.29 & 0.88 & -2.01 & 1.42 & 1.00 \\
\hline zSPQDis:relation & -0.50 & 0.95 & -2.39 & 1.36 & 1.00 \\
\hline soa:relation & -0.51 & 0.70 & -1.90 & 0.88 & 1.00 \\
\hline directness:zSPQPos:soa & -1.75 & 2.00 & -5.67 & 2.22 & 1.00 \\
\hline directness:zSPQNeg:soa & -0.80 & 2.13 & -5.05 & 3.41 & 1.00 \\
\hline directness:zSPQDis:soa & 0.57 & 2.25 & -3.88 & 4.98 & 1.00 \\
\hline directness:zSPQPos:relation & -0.51 & 0.82 & -2.11 & 1.12 & 1.00 \\
\hline directness:zSPQNeg:relation & -0.58 & 0.87 & -2.29 & 1.13 & 1.00 \\
\hline directness:zSPQDis:relation & 0.64 & 0.94 & -1.18 & 2.48 & 1.00 \\
\hline directness:soa:relation & -0.92 & 0.67 & -2.23 & 0.38 & 1.00 \\
\hline zSPQPos:soa:relation & -1.02 & 0.71 & -2.40 & 0.40 & 1.00 \\
\hline zSPQNeg:soa:relation & -0.38 & 0.76 & -1.87 & 1.12 & 1.00 \\
\hline zSPQDis:soa:relation & 0.11 & 0.82 & -1.50 & 1.72 & 1.00 \\
\hline directness:zSPQPos:soa:relation & -0.85 & 0.72 & -2.26 & 0.56 & 1.00 \\
\hline directness:zSPQNeg:soa:relation & -0.55 & 0.76 & -2.06 & 0.93 & 1.00 \\
\hline directness:zSPQDis:soa:relation & 0.37 & 0.82 & -1.24 & 1.98 & 1.00 \\
\hline sigma & 49.47 & 0.55 & 48.40 & 50.55 & 1.00 \\
\hline beta & 141.50 & 0.98 & 139.57 & 143.42 & 1.00 \\
\hline
\end{tabular}


Table 3 (on next page)

OLIFE model summary of population-level effects estimates 
parameter mean
SE lower

bound upper

bound

Rhat

\begin{tabular}{|c|c|c|c|c|c|}
\hline Intercept & 614.98 & 5.19 & 604.72 & 624.91 & 1.00 \\
\hline directness1 & 8.27 & 4.64 & -0.87 & 17.32 & 1.00 \\
\hline zOLIFEPos & -0.27 & 4.72 & -9.53 & 9.07 & 1.00 \\
\hline zOLIFENeg & -1.74 & 4.59 & -10.81 & 7.13 & 1.00 \\
\hline zOLIFEDis & 0.84 & 4.89 & -8.73 & 10.42 & 1.00 \\
\hline zOLIFEImp & 5.56 & 4.98 & -4.30 & 15.29 & 1.00 \\
\hline soa1 & 7.92 & 1.81 & 4.38 & 11.49 & 1.00 \\
\hline relation1 & -7.30 & 0.87 & -9.02 & -5.58 & 1.00 \\
\hline directness1:zOLIFEPos & -0.22 & 4.75 & -9.62 & 9.07 & 1.00 \\
\hline directness1:zOLIFENeg & -1.72 & 4.69 & -10.83 & 7.41 & 1.00 \\
\hline directness1:zOLIFEDis & 4.52 & 4.96 & -5.20 & 14.22 & 1.00 \\
\hline directness1:zOLIFEImp & -0.09 & 4.93 & -9.59 & 9.55 & 1.00 \\
\hline directness1:soa1 & 0.81 & 1.82 & -2.78 & 4.37 & 1.00 \\
\hline zOLIFEPos:soa1 & 0.25 & 1.93 & -3.51 & 4.03 & 1.00 \\
\hline zOLIFENeg:soa1 & 1.22 & 1.88 & -2.45 & 4.86 & 1.00 \\
\hline zOLIFEDis:soa1 & -1.05 & 2.11 & -5.19 & 3.08 & 1.00 \\
\hline zOLIFEImp:soa1 & 5.00 & 2.08 & 0.91 & 9.12 & 1.00 \\
\hline directness1:relation1 & -4.64 & 0.79 & -6.21 & -3.08 & 1.00 \\
\hline zOLIFEPos:relation1 & 0.52 & 0.80 & -1.05 & 2.07 & 1.00 \\
\hline zOLIFENeg:relation1 & -0.45 & 0.77 & -1.96 & 1.07 & 1.00 \\
\hline zOLIFEDis:relation1 & -1.01 & 0.88 & -2.74 & 0.71 & 1.00 \\
\hline zOLIFEImp:relation1 & 0.50 & 0.85 & -1.15 & 2.17 & 1.00 \\
\hline soa1:relation1 & -0.28 & 0.71 & -1.64 & 1.10 & 1.00 \\
\hline directness1:zOLIFEPos:soa1 & 1.06 & 1.91 & -2.71 & 4.84 & 1.00 \\
\hline directness1:zOLIFENeg:soa1 & -0.39 & 1.89 & -4.10 & 3.36 & 1.00 \\
\hline directness1:zOLIFEDis:soa1 & 1.09 & 2.07 & -2.93 & 5.20 & 1.00 \\
\hline directness1:zOLIFEImp:soa1 & -0.87 & 2.05 & -4.91 & 3.18 & 1.00 \\
\hline directness1:zOLIFEPos:relation1 & -0.23 & 0.79 & -1.77 & 1.32 & 1.00 \\
\hline directness1:zOLIFENeg:relation1 & -0.56 & 0.77 & -2.08 & 0.94 & 1.00 \\
\hline directness1:zOLIFEDis:relation1 & -0.89 & 0.87 & -2.58 & 0.82 & 1.00 \\
\hline directness1:zOLIFEImp:relation1 & 2.12 & 0.84 & 0.47 & 3.76 & 1.00 \\
\hline directness1:soa1:relation1 & -0.85 & 0.67 & -2.15 & 0.47 & 1.00 \\
\hline zOLIFEPos:soa1:relation1 & -0.30 & 0.71 & -1.69 & 1.07 & 1.00 \\
\hline zOLIFENeg:soa1:relation1 & -0.43 & 0.69 & -1.80 & 0.92 & 1.00 \\
\hline
\end{tabular}




$\begin{array}{lccccc}\text { zOLIFEDis:soa1:relation1 } & -0.25 & 0.78 & -1.80 & 1.26 & 1.00 \\ \text { zOLIFEImp:soa1:relation1 } & 0.32 & 0.75 & -1.15 & 1.82 & 1.00 \\ \text { directness1:zOLIFEPos:soa1:relation1 } & 0.60 & 0.71 & -0.80 & 2.01 & 1.00 \\ \text { directness1:zOLIFENeg:soa1:relation1 } & -0.79 & 0.69 & -2.15 & 0.56 & 1.00 \\ \text { directness1:zOLIFEDis:soa1:relation1 } & -0.82 & 0.78 & -2.32 & 0.73 & 1.00 \\ \text { directness1:zOLIFEImp:soa1:relation1 } & -0.17 & 0.75 & -1.64 & 1.34 & 1.00 \\ \text { sigma } & 49.41 & 0.55 & 48.32 & 50.49 & 1.00 \\ \text { beta } & 141.45 & 0.98 & 139.52 & 143.39 & 1.00 \\ & & & & & \end{array}$

\section{1}

